\title{
ARTICLE
}

Received 7 Feb 2013 | Accepted 24 Apr 2013 | Published 10 Jun 2013 DOl: 10.1038/ncomms2927

\section{Caspase- 2 is required for dendritic spine and behavioural alterations in J20 APP transgenic mice}

\author{
Julio Pozueta ${ }^{1, \star}$, Roger Lefort ${ }^{1, \star}$, Elena M. Ribe ${ }^{1, \dagger}$, Carol M. Troy ${ }^{1}$, Ottavio Arancio ${ }^{1} \&$ Michael Shelanski
}

Caspases have critical roles in Alzheimer's disease pathogenesis. Here we show that caspase- 2 is required for the cognitive decline seen in human amyloid precursor protein transgenic mice (J20). The age-related changes in behaviour and dendritic spine density observed in these mice are absent when they lack caspase-2, in spite of similar levels of amyloid beta $(A \beta)$ deposition and inflammation. A similar degree of protection is observed in cultured hippocampal neurons lacking caspase-2, which are immune to the synaptotoxic effects of $A \beta$. Our studies suggest that caspase- 2 is a critical mediator in the activation of the RhoA/ROCK-II signalling pathway, leading to the collapse of dendritic spines. We propose that this is controlled by an inactive caspase-2/RhoA/ROCK-II complex localized in dendrites, which dissociates in the presence of $A \beta$, allowing for their activation and entry in the spine. These findings directly implicate caspase-2 as key driver of synaptic dysfunction in Alzheimer's disease and offer novel therapeutic targets.

\footnotetext{
${ }^{1}$ Department of Pathology and Cell Biology and The Taub Institute for Research on Alzheimer's Disease and the Aging Brain v, Columbia University, New York, New York 10032, USA. * These authors contributed equally to this work. † Present address: Division of Psychological Medicine and Old Age Psychiatry, Institute of Psychiatry, King's College London, London SE5 8AF, UK. Correspondence and requests for materials should be addressed to M.S. (email: mls7@columbia.edu).
} 
$\mathrm{M}$ ice that carry transgenes encoding mutant human amyloid precursor protein (hAPP) develop many of the changes associated with Alzheimer's disease (AD), including deficits in learning and memory, inhibition of longterm potentiation, loss of dendritic spines, profuse amyloid plaques and increased phosphorylation of the tau protein ${ }^{1}$. Changes similar to those seen in transgenic animals are replicated in vitro by treatment of cultured primary neurons with aggregated soluble amyloid beta (A $\beta$ ) oligomers, or in vivo by direct injection of $A \beta$ oligomers into the hippocampus ${ }^{2,3}$. As neuronal death is usually not observed in hAPP transgenic mice, the non-apoptotic role of caspases (cysteine-aspartic proteases), in driving ADrelated changes have remained largely unexplained. However, it is clear that caspases are crucial in contexts other than apoptosis, particularly in the maintenance of normal synaptic functions, whether in sculpting dendritic arborization and synapses ${ }^{4,5}$, guiding and pruning axons $s^{6,7}$, or in regulating long-term potentiation and long-term depression ${ }^{8,9}$.

Disruption of normal synaptic function is one of the earliest events in $A D^{10,11}$, raising the intriguing possibility that aberrant activation of caspases in $\mathrm{AD}$ brains may also have a pathogenic non-apoptotic role in driving these synaptic changes. Several caspases have been shown to be activated in human and in $\mathrm{AD}$ mouse model brains, including caspase-1, caspase-2, caspase-3, caspase- 6 and caspase-9 (refs 12-16). Of all these caspases, however, our work has shown that only caspase- 2 appears to be required for the apoptotic effects of $A \beta$ in cultured hippocampal neurons. Hippocampal neurons for caspase- 2 null mice are resistant to $A \beta$ in spite of elevated levels of caspase-3, caspase- 9 and the pro-apoptotic protein Smac/Diablo ${ }^{17,18}$

In the current study, we have revisited these earlier findings and investigated whether caspase- 2 also has a significant role in mediating the effects of $A \beta$ on synaptic plasticity and memory. We approached this question first in the J20 transgenic $A D$ mouse model ${ }^{19}$. This mouse model features elevated levels of $A \beta 42$, resulting from the introduction of the Swedish (K670N/ M671L) and Indiana (V717F) hAPP mutations (that is, hAPPSwInd) and develop numerous plaques by the age of 5-7 months, as well as an age-dependent decline in learning in memory. We show that crossing the J20 mice with caspase- 2 null mice results in the complete prevention of the memory impairments, as assessed by the radial-arm water maze (RAWM), and prevention of the loss of dendritic spine density typically observed in J20 mice, without significantly affecting amyloid load, inflammation and neuritic dystrophy. We further validated these results in an in vitro $\mathrm{AD}$ model using cultured primary hippocampal neurons where we show that downregulation of caspase- 2 blocks the $A \beta$-mediated changes in dendritic spines. Additionally, our data suggest that caspase-2 may be a critical component in the activation and signalling of the Rho-GTPase RhoA, a critical regulator of dendritic spine morphology ${ }^{20,21}$. Taken together, these studies suggest that caspase-2 has a critical role in mediating the synaptic changes and memory alteration induced by $\mathrm{A} \beta$ in $\mathrm{AD}$, and highlight caspase- 2 as a potential target for $\mathrm{AD}$ therapy.

\section{Results}

Synaptotoxic effects of $\mathbf{A} \boldsymbol{\beta}$ requires caspase- 2 . In order to determine if caspase- 2 has a role in the subapoptotic effects of oligomeric $\mathrm{A} \beta$ at low (nM range) concentrations, in addition to its apoptotic role at higher $(\mu \mathrm{M}$ range) doses (Supplementary Fig. S1a), we exposed hippocampal neurons from wild-type (WT) rats to $300 \mathrm{nM} \mathrm{A} \beta$ with and without downregulation of caspase- 2 and examined the effects on dendritic spine density and on levels of RhoA-GTP and ROCK-II. This resulted in a progressive decrease in spine density over the initial $24 \mathrm{~h}$ of exposure as well as increases in the levels of active RhoA-GTP and ROCK-II (Fig. 1a,b and Supplementary Fig. S1b,c). These effects were not seen in neurons exposed to the reverse A $\beta$ peptide $42-1$ (Supplementary Fig. S2). When caspase-2 levels were decreased with an small interfering RNA (siRNA) by 65\% (Fig. 1f), there was a partial blockade of the effect of $A \beta$ on spine density and the levels of RhoA-GTP and ROCK-II, and complete protection against $A \beta$-induced spine reduction (Fig. 1c-e,g). Downregulation of caspase- 3 , caspase- 8 and caspase- 9 individually had no effect on spine density or on the levels of RhoA-GTP (Fig. 2a,b,e-g). As siRNA-mediated downregulation of each caspases was not complete (Fig. 2f), we wanted to rule out the possibility that residual caspase activity was responsible for the lack of protection. For that, we used commercially available irreversible caspase inhibitors to block caspase activity. Consistent with our siRNA results, only the caspase-2 inhibitor (VDVAD) blocked the effects of $A \beta$ on dendritic spines, while the caspase- 3 (DEVD) and caspase-8 (LEHD) inhibitors had no significant protective effect (Fig. 2c,d). These results were confirmed using hippocampal neurons from WT and caspase- 2 null mice (Casp2KO). Before $A \beta$ treatment, the spine density in neurons from each mouse line was similar. However, when the neurons were treated with $A \beta$, a significant loss of spines $(\sim 50 \%$ after $24 \mathrm{~h}$ ) was seen in WT neurons but not in those derived from the Casp2KO mice (Fig. 1h). These experiments suggest that caspase2 is not only critical for $A \beta$-induced apoptosis but also for the effects of $A \beta$ on dendritic spines in cultured neurons.

Memory impairments are absent in $\mathbf{J} 20$ mice lacking caspase-2. As experiments in cell culture are acute and cannot provide data on chronic effects or on the relationship of these changes to alterations in memory, we generated a new transgenic mouse line by crossing J20 mice ${ }^{19}$ that overexpress mutant hAPP carrying the Swedish (K670N/M671L) and Indiana (V717F) hAPP mutations (that is, hAPPSwInd), with caspase-2 knock-out (Casp2KO) mice ${ }^{22}$, with both parental lines being on C57BL/6 backgrounds. Four distinct lines with four different genotypes were derived from these crosses: hAPP $-/-/ \mathrm{Casp} 2^{+/}+$referred to as WT; $\mathrm{hAPP}^{+/-} / \mathrm{Casp}^{+/+}$referred to as J20; $\mathrm{hAPP}^{+1-} /$ Casp2 ${ }^{-1-}$ referred to as J20/Casp2KO and hAPP ${ }^{-/-} / \mathrm{Casp}^{-1-}$ referred to as Casp2KO. We analysed these mice for dendritic spine morphology, amyloid accumulation, astrocytosis, microgliosis, dystrophic neurites and behaviour, as measured by RAWM.

The animals were tested in the RAWM at 4, 9 and 14 months of age and then killed. The brains were examined by diOlistic labelling to determine dendritic spine density and the levels of active RhoA-GTP and ROCK-II were measured by western blotting. As differences in behaviour or in dendritic spine density could simply reflect differences in $A \beta$ load or deposition, sections were also stained for plaques with an $A \beta$-specific antibody (6E10), and levels of soluble and acid-extractable $A \beta$ were measured at each of the ages. As the results at 9 and 14 months were quite similar, we will focus our discussion on the changes at 14 months.

At 4 months of age there were no detectable amyloid plaques in the brains of any of the 4 mouse lines and extractable $A \beta$ levels showed no significant differences. No significant difference in task acquisition in RAWM was seen among the lines at 4 or 9 months of age (Fig. 3a,b). On the contrary, at 14 months of age the J20 animals and the Casp2KO animals were slower to learn the task than either WT or J20/Casp2KO animals (Fig. 3c). More importantly, only the J20 animals were impaired in task retention when compared with the three other lines at 4,9 and 14 months of age (Fig. 3d-f). The differences in task retention were 
a
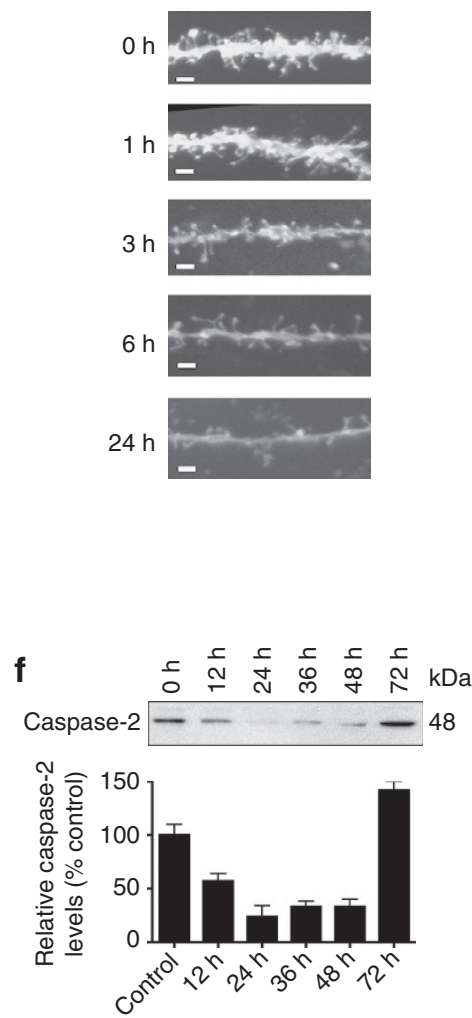

b
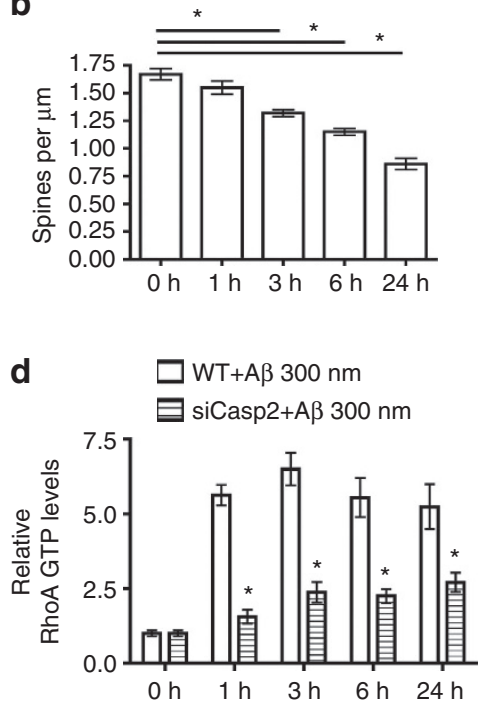

g

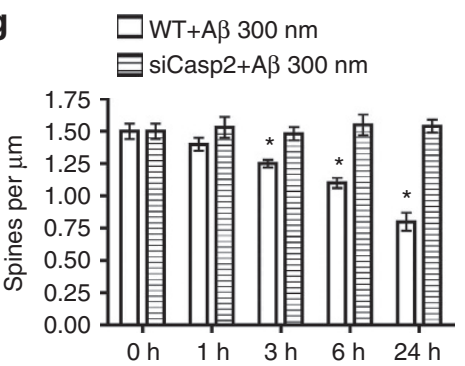

C

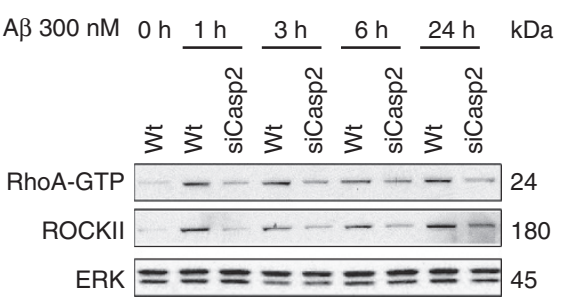

e

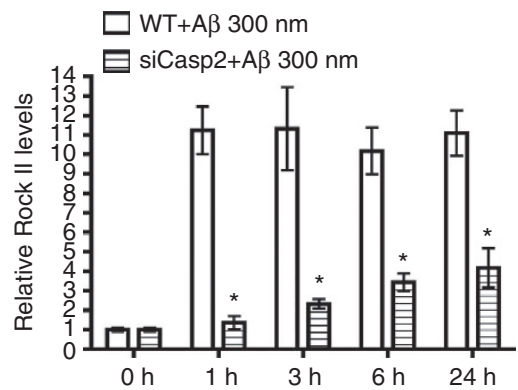

h

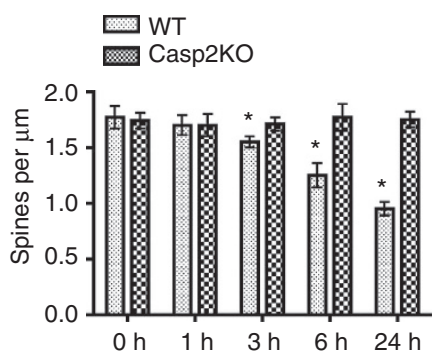

Figure 1 | Caspase-2 is required for A $\boldsymbol{\beta}$-induced dendritic spine loss in hippocampal primary cultures. (a) Representative images of labelled dendrites over the course of $24 \mathrm{~h}$ after treatment with $300 \mathrm{nM} \mathrm{A \beta}$. (b) Spine density (spines per $\mu \mathrm{m}$ ) changes in rat primary hippocampal cultures treated with $300 \mathrm{nM} \mathrm{A} \beta$ for $24 \mathrm{~h}$. Data presented as mean \pm s.e.m. ( $n=3$ independent experiments, 150 dendrites per point, ${ }^{\star} P<0.05$, one-way analysis of variance (ANOVA)). (c) Western blot analysis of primary hippocampal cultures pretreated for $12 \mathrm{~h}$ with $80 \mathrm{nM}$ of Pen1-siRNA against caspase-2 showing no changes in RhoA-GTP or ROCK-II after a 24-h treatment with $300 \mathrm{nM} \mathrm{A \beta}$. (d) Densitometric quantification of RhoA-GTP immunoblots. Data presented as the mean \pm s.e.m. ( $n=3$ independent experiments, ${ }^{\star} P<0.05$, two-tailed Student's $t$-test). (e) Densitometric quantification of ROCK-II immunoblots. Data presented as the mean \pm s.e.m. ( $n=3$ independent experiments, ${ }^{\star} P<0.05$, two-tailed Student's $t$-test). (f) Rat hippocampal primary cultures treated with $80 \mathrm{nM}$ Pen1-siRNA against caspase-2 showing a time-dependent decrease in caspase-2 levels. Representative immunoblot for caspase-2 levels. Densitometric analysis of caspase-2 levels showing a $60 \%$ reduction by $12 \mathrm{~h}$ and persisting over $48 \mathrm{~h}$. (g) Rat hippocampal primary cultures pretreated for $24 \mathrm{~h}$ with $80 \mathrm{nM}$ Pen1-siRNA against caspase-2 show no decrease in spine density after treatment with $300 \mathrm{nM} A \beta$. Data presented as the mean \pm s.e.m. ( $n=3$ independent experiments, 150 dendrites per point, ${ }^{\star} P<0.05$, one-way ANOVA). (h) Hippocampal primary cultures derived from

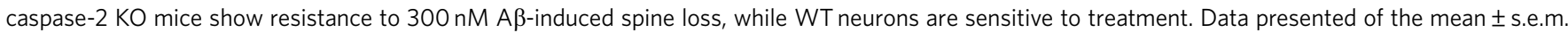
( $n=3$ independent experiments, 150 dendrites per point, ${ }^{\star} P<0.05$, one-way ANOVA).

independent of motor, sensory or motivational changes among the different lines, as there were no observable changes in the latency to find a visible platform or in the swimming speed of these mice (Fig. 3g,h).

Synaptic changes are abrogated in $\mathbf{J} 20$ mice lacking caspase-2. Immediately after the behavioural analyses described above, the mice were killed to evaluate whether the differences in behaviour were reflected by changes in dendritic spine density and morphology. Spine density did not differ greatly at 4 months of age among genotypes (Fig. 4a). Moreover, there were no significant alterations in PSD95, Synapsin-1, RhoA-GTP or ROCK-II at 4 months of age (Supplementary Fig. S3a,b). However, analysis of spine morphology at this age revealed a significant decrease in the mushroom subtype in the J20 mice as compared with the WT and the J20/Casp2KO mice. The decrease in mushroom spines in the J20 animals is correlated with an increase in the stubby subtype that was not seen in the other lines (Fig. 4c-e). When similar studies were carried out at 14 months of age, dendritic spine density in the J20 animals was reduced to $\sim 50 \%$ of the level seen in WT animals while the J20/Casp2KO animals, and the Casp2KO animals showed no loss of dendritic spines (Fig. 4a,b). To determine if these changes were limited to the hippocampus, we examined the frontal cortex where we found similar spine changes (Supplementary Fig. S3c). Similarly, biochemical analysis of the hippocampus at 14 months showed a reduction in both PSD95 and Synapsin-1 and an increase in RhoA-GTP and ROCK-II in the J20 animals (Fig. 4f,g). No changes in these markers were seen in the other three lines.

At 14 months of age, both the J20 and J20/Casp2KO animals showed approximately equal accumulation of amyloid plaques (Fig. 5a,b) and equal levels of expression of hAPP (Fig. 5d). No plaques were seen in the WT and Casp2KO animals and no hAPP expression was found. Levels of soluble and formic acidextractable A $\beta$ did not differ significantly between J20 and J20/ Casp2KO mice (Fig. 5e). Although differences did not meet statistical significance, $A \beta$ levels appeared be marginally lower in 
a

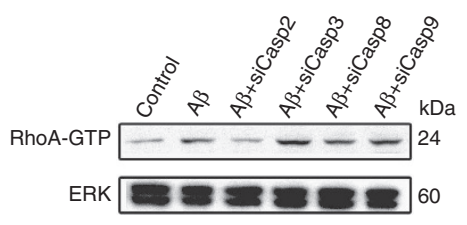

C

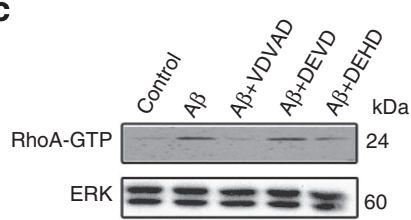

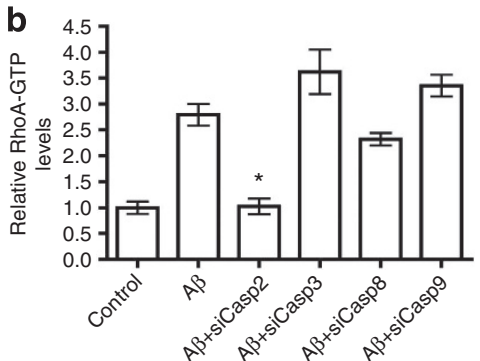

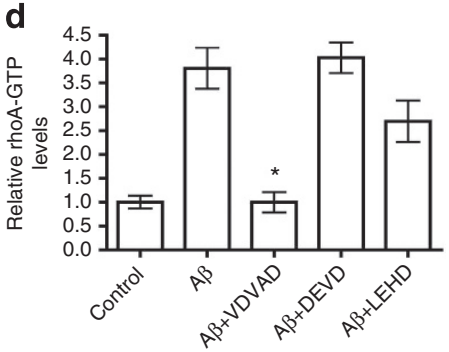

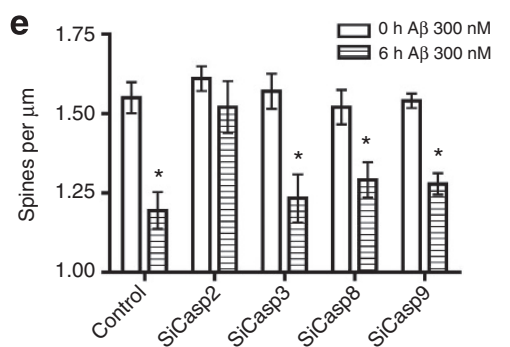

f
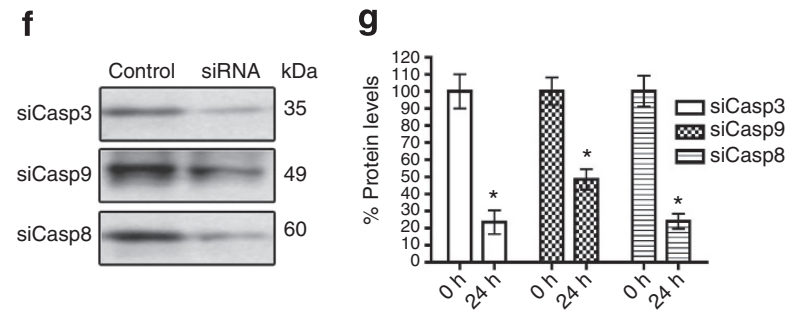

Figure 2 | Downregulation or inactivation of caspase-2 blocks A $\beta$-mediated effects on primary hippocampal cultures. (a) Representative western blot of hippocampal primary cultures treated for $1 \mathrm{~h}$ with $300 \mathrm{nM} \mathrm{A} \beta$. The increase in RhoA-GTP levels is reversed by pretreatment with $80 \mathrm{nM}$ Pen1-siRNA against caspase-2 but not with siRNA against caspase-3 (siCasp3), -8 (siCasp8) or -9 (siCasp9). (b) Densitometric analysis of RhoA-GTP levels from the immunoblots showing only the siCasp2 is able to block the changes observed from A $\beta$. Data presented as the mean \pm s.e.m. ( $n=3$ independent experiments, ${ }^{\star} P<0.05$, two-tailed Student's $t$-test). (c) Representative immunoblot from hippocampal primary cultures treated for $1 \mathrm{~h}$ with $300 \mathrm{nM} A \beta$; cultures were pretreated for 30 min with $0.5 \mu \mathrm{M}$ of each caspase inhibitors: VDVAD (caspase-2); DEVD (caspase-3/7); LEHD (caspase-9).

(d) Densitometric representation of RhoA-GTP levels of the immunoblot in c. Only caspase-2 inhibitor VDVAD blocks the A $\beta$-induced increase in RhoA-GTP. Data presented as the mean \pm s.e.m. ( $n=3$ independent experiments, ${ }^{\star} P<0.05$, two-tailed Student's $t$-test). (e) Spine density in primary hippocampal cultures pretreated for $24 \mathrm{~h}$ with siRNAs against caspase- $2,-3,-8$ and -9 and then treated for $6 \mathrm{~h}$ with $300 \mathrm{nM}$ A $\beta$. Only siCasp2 protects against $A \beta$-mediated spine changes. Data presented as the mean \pm s.e.m. ( $n=3$ independent experiments, 50 different dendrites per point; ${ }^{\star} P<0.05$, one-way analysis of variance). (f) Representative immunoblots of primary cultures treated for $24 \mathrm{~h}$ with the respective siRNAs. (g) Densitometric analysis of caspase- $3,-8$ and -9 levels after $24 \mathrm{~h}$ treatment with the siRNA.

the J20/Casp2KO animals. GFAP staining revealed similar increases in astrocytosis both in the J20 and J20/Casp2KO as compared with the WT and Casp2KO animals (Fig. 5a,c). Increased tau phosphorylation is seen in the J20 animals but not in the J20/Casp2KO animals (Supplementary Fig. S3d). Staining for dystrophic neurites with SMI-312 (refs 23,24) and for microglia with Iba-1 (ref. 25) showed no significant difference between the J20 and J20/Casp2KO mice in the area surrounding the amyloid plaques (Supplementary Fig. S4a-c).

Caspase-2 and inactive RhoA form a complex. It has been demonstrated that $\mathrm{A} \beta$-treatment of neurons activates caspase- 2 (ref. 26). The results presented above suggest that caspase- 2 might have a role in the regulation of dendritic spine dynamics through RhoA. It has been reported that RhoB, a protein that is over $90 \%$ homologous with RhoA, is capable of binding to caspase-2 (ref. 27). To determine if a similar interaction underlies the role of caspase- 2 in $A \beta$-mediated synaptic changes, we examined the interaction of these proteins in a series of co-immunoprecipitation studies from lysates of primary hippocampal neurons. We found that inactive RhoA (RhoA-GDP) but not active RhoA (RhoA-GTP) co-precipitated with caspase-2 (Fig. 6a). We were unable to directly immunoprecipitate RhoA-GDP or RhoA-GTP with the antibodies at our disposal and therefore turned to a cell-free system using recombinant caspase-2 and recombinant His-tagged inactive RhoA [His-RhoA] and Histagged constitutively active RhoA [His-RhoA(Q63L)]. Consistent with our in vitro co-immunoprecipitation studies, only His-RhoA co-precipitated with caspase-2, but not constitutively active RhoA [His-RhoA(Q63L)]. Conversely, caspase-2 co-precipitated only with inactive His-RhoA, and not with active His-RhoA(Q63L) (Fig. 6b,c). Together, these results suggest a direct interaction between caspase- 2 and inactive RhoA and not with active RhoA. This interaction does not appear to inhibit the activity of caspase-2, as the addition of inactive RhoA to recombinant active caspase- 2 failed to block its activity as measured by fluorometric assay (Supplementary Fig. S5a).

Synaptotoxic effects of $\mathrm{A} \boldsymbol{\beta}$ are RhoA/ROCK-II dependent. Interestingly, we found that the activity of caspase- 2 may be 


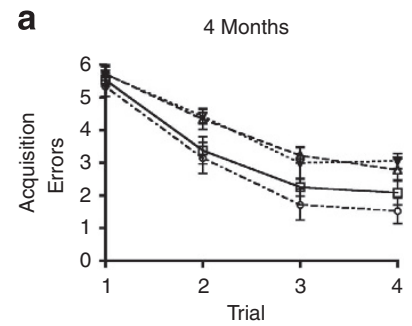

b
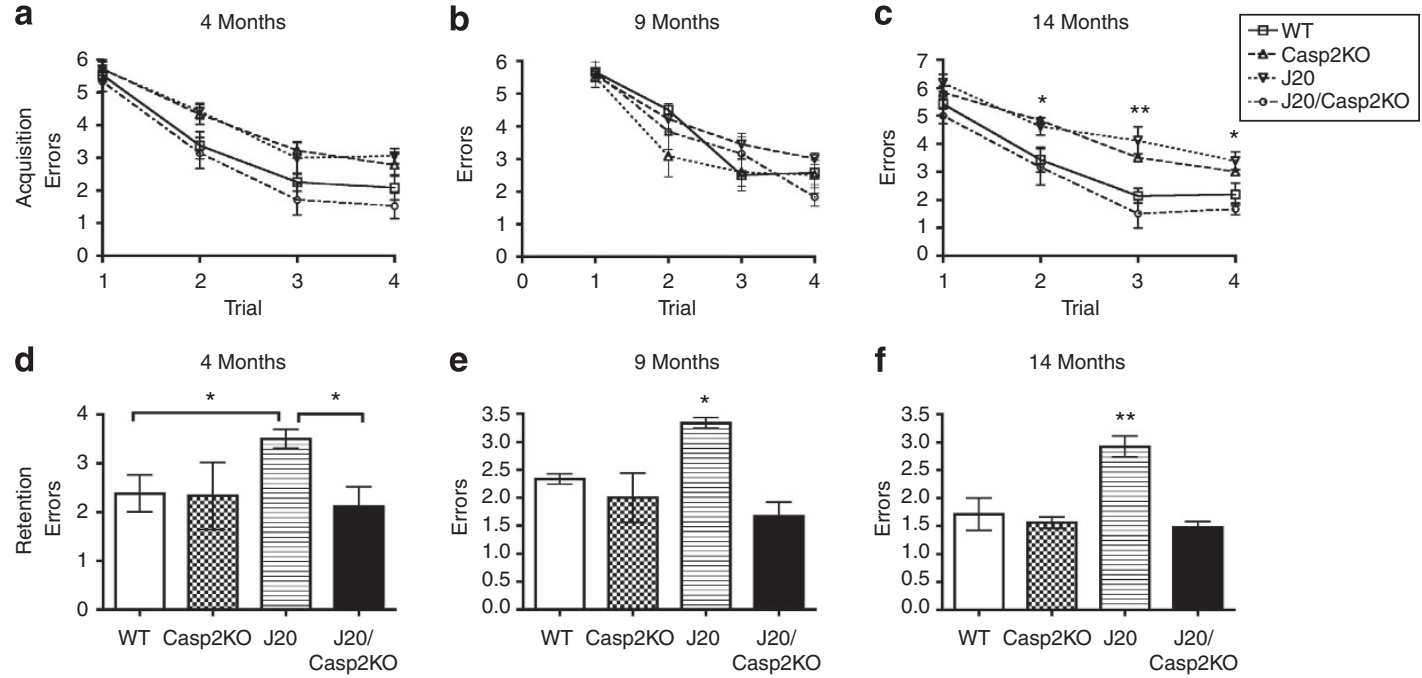

e

f
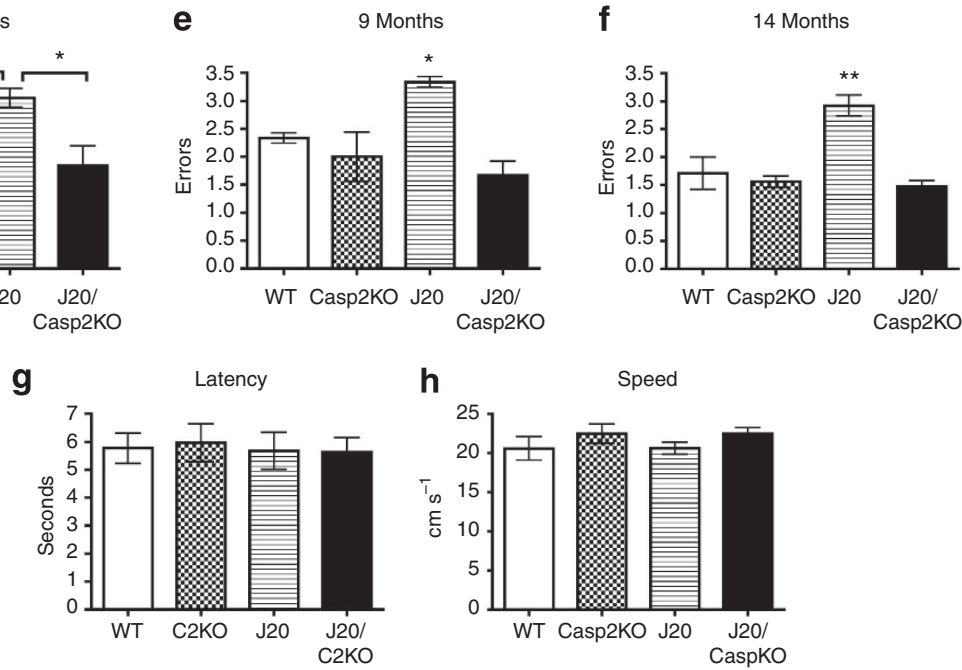

Figure 3 | J20/Casp2KO mice lack memory deficits seen in J20 mice. Mice from the four different lines were tested in the RAWM for spatial working memory at the ages of 4, 9 and 14 months. (a) At 4 months, there was no significant difference in task acquisition between WT, Casp2KO, J2O and J20/Casp2KO mice. (b) At 9 months, there was no significant difference in task acquisition between WT, Casp2KO, J20 and J20/Casp2KO. (c) At 14 months, J2O and Casp2KO mice showed impaired acquisition of the task as compared with WT and J20/Casp2KO littermates (7 male mice per group ${ }^{\star} P<0.05$, two-way analysis of variance (ANOVA)). (d) During the retention trial, 4-month-old J20 mice showed a memory deficit compared with WT and J20/Casp2KO littermates, but not with Casp2KO (7 male mice per group, ${ }^{\star} P<0.05$, two-way ANOVA). (e) At 9 months, J20 mice showed memory deficits compared with the other three lines: WT, Casp2KO and J20/Casp2KO (7 male mice per group, ${ }^{\star} P<0.05$, two-way ANOVA). (f) Memory deficits persisted at 14 months of age $\left({ }^{\star} P<0.05,{ }^{\star} P<<0.01\right.$, two-way ANOVA). (g,h) The four lines performed similarly in visible platform trials with regard to time to reach the platform (e) and speed (f).

critical in mediating the effects of $A \beta$ on cytoskeletal dynamics through its recently reported role in cleaving and activating the RhoA effector ROCK-II ${ }^{28}$. Indeed, exposure of primary hippocampal neurons to $A \beta$ resulted in increased ROCK-II cleavage, generating a $\sim 130 \mathrm{kDa}$ fragment (Supplementary Fig. S6a,b), consistent with previous reports ${ }^{28,29}$. Depletion of caspase-2 in these neurons before $A \beta$ treatment blocked ROCK-II cleavage, confirming the role of caspase-2 in cleaving ROCK-II.

If RhoA and ROCK-II have critical roles in $A \beta$-mediated dendritic spine alterations, we would predict that inhibition of their activities should be able to block these changes. When primary hippocampal neuronal cultures were pretreated with the RhoA inhibitor, C3 transferase, or the ROCK-II inhibitor, Y27632, and then exposed to $A \beta$, the changes in spine density were abrogated (Fig. 6d,e). To determine if caspase-2 was involved more broadly in the control of RhoA-mediated signalling. We treated cells for $1 \mathrm{~h}$ with lysophosphatidic acid leading to the large increase in RhoA-GTP. Pretreatment of the cells with either siCasp2 or the commercially available caspase-2 inhibitor VDVAD partially blocks the increase in RhoA-GTP suggesting that caspase- 2 is involved in regulating RhoA in response to diverse stimuli (Supplementary Fig. S5b,c).

Caspase-2 regulates RhoA localization. There is evidence that suggests that the distribution of RhoA may to be altered in $\mathrm{AD}$ and in $\mathrm{AD}$ mouse models ${ }^{30}$. We wondered whether $\mathrm{A} \beta$ could affect the localization of RhoA in neurons and whether caspase-2 has a role in this process. We exposed primary hippocampal neurons to $A \beta$ and measured the localization of PSD95, RhoA and caspase- 2 over the course of $24 \mathrm{~h}$. (Fig. 7a,b,f,g). Before treatment with $\mathrm{A} \beta$ we found very low levels $(\sim 10 \%)$ of colocalization of RhoA with the synaptic marker PSD95 (Fig. 7a,c), suggesting that the majority of RhoA is found outside dendritic spines. After treatment with $A \beta$, there was a significant increase $(\sim 20 \%)$ after $6 \mathrm{~h}$ in the colocalization of RhoA and ROCK-II with PSD95, suggesting that $A \beta$ treatment results in the recruitment of these proteins to dendritic spines, a translocation that has been suggested for RhoA in response to glutamate receptor modulation ${ }^{33}$. Although the ROCK-II increase at the spine is significant by $1 \mathrm{~h}$ and remains elevated over the treatment period, the RhoA translocation is more gradual. Similarly, before treatment, caspase- 2 was mostly present in the dendritic shaft (Fig. 7a,f), but after treatment with $A \beta$, we observed a clear increase $(\sim 10 \%)$ in caspase-2 colocalization with PSD95 between 1 and $6 \mathrm{~h}$ post-treatment (Fig. $7 \mathrm{~d}$ ), suggesting that $A \beta$ also triggers the translocation of caspase- 2 to dendritic spines. When caspase-2 was downregulated in neurons before treatment with $A \beta$, the colocalization of RhoA and PSD95 was significantly diminished (Fig. 7f,g), consistent with a role for caspase- 2 as a facilitator of RhoA translocation to dendritic spines after $A \beta$ treatment. $A \beta$ treatment also resulted in increased colocalization of caspase-2 with both RhoA and ROCK-II 
a

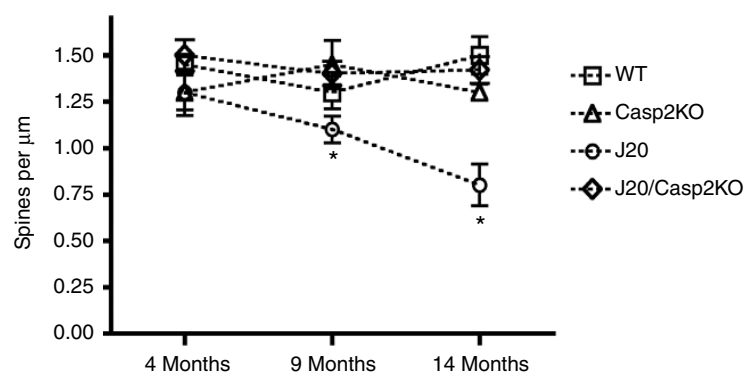

b

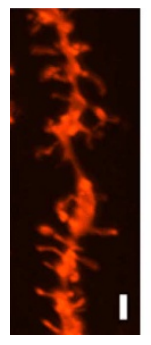

WT

d

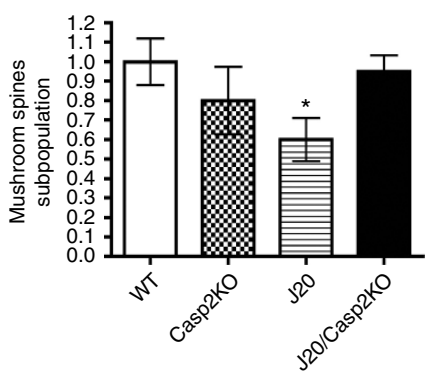

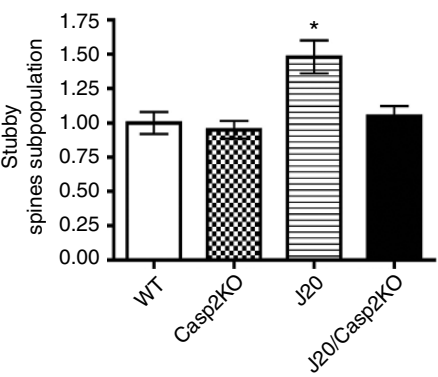

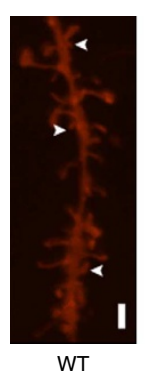

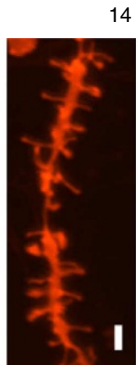

Casp2KO
14 Months

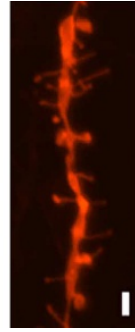

J20

4 Months

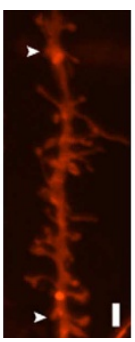

Casp2KO

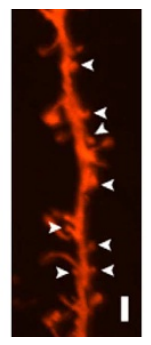

J20

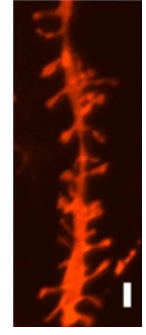

J20/Casp2KO f

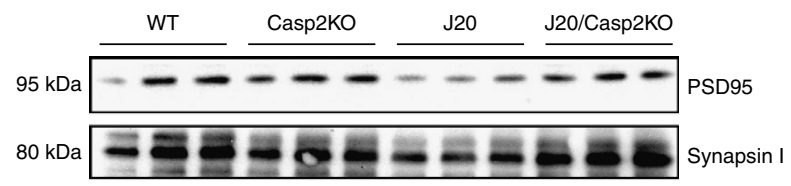

$180 \mathrm{kDa}$ - $-\ldots-\ldots$ ROCKII

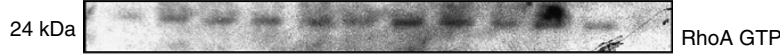

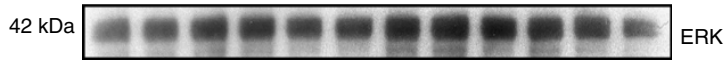

g

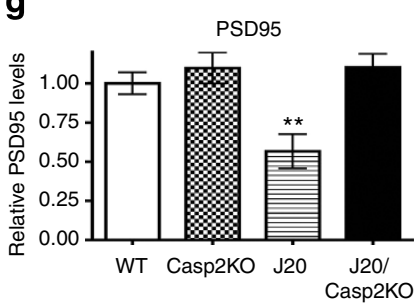

ROCKII

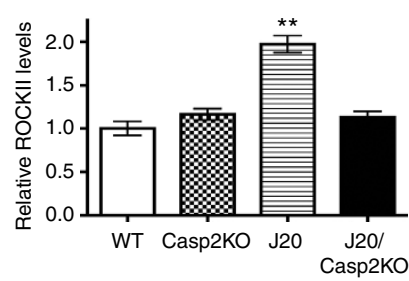

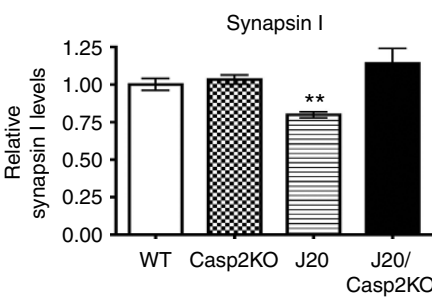

RhoA GTP

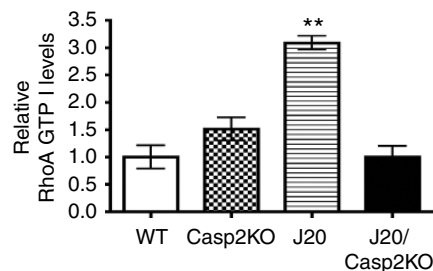

Figure 4 | Dendritic spine loss in J20 mice is reversed in J20/Casp2KO animals. Mice from each line (WT, Casp2KO, J20 and J20/Casp2KO) were analysed at 4, 9 and 14 months of age for spine changes. (a) J20 mice showed a significant decrease in spine density at 9 and 14 months of age compared with WT, Casp2KO and J20/Casp2KO mice. Data presented as the mean spine density \pm s.e.m. $\left(n=6\right.$ mice, 50 dendrites per mouse; ${ }^{\star} P<0.05$, two-way analysis of variance (ANOVA)). (b) Images of Dil-labelled dendrites and spine morphologies in hippocampal neurons from 14-month-old mice of each line. (c) J20 mice showed a significant decrease in mushroom spine subpopulation at 4 months of age, while J20/Casp2KO mice showed same levels as WT. Histogram showing the relative number of mushroom-type spines compared with WT mice. Data presented as the mean density \pm s.e.m. ( $n=5$ mice, 50 dendrites per mouse, ${ }^{\star} P<0.05$, two-way ANOVA). (d) J20 mice showed a significant increase in stubby spine subpopulation at 4 months of age, while J20/Casp2KO mice showed similar levels as WT. Histogram showing the relative density of stubby-type spines compared with WT mice. Data presented as the mean density \pm s.e.m. ( $n=5$ mice, 50 dendrites per mouse, ${ }^{\star} P<0.05$, two-way ANOVA). (e) Images of Dil-labelled dendrites and spine morphologies in hippocampal neurons from 4-month-old mice of each line. (f) Representative western blots of hippocampal extracts from 14-month-old mice showing that alterations in synaptic and dendritic spine markers seen in J20 mice are absent in J20/Casp2KO mice $\left(n=10\right.$ mice, ${ }^{\star \star} P<0.01$, two-tailed Student's $t$-test). (g) Densitometric quantification of immunoblots in c. Levels of post- and pre-synaptic markers PSD95 and Synapsin-1 were reduced in $\mathrm{J} 20$ mice compared with WT and J20/Casp2KO mice ( ${ }^{\star \star} P<0.01$, two-tailed Student's $t$-test). Levels of ROCK-II and RhoA-GTP proteins were elevated in $\mathrm{J} 20$ mice compared with WT and J20/Casp2KO mice ( ${ }^{\star \star} P<0.01$, two-tailed Student's $t$-test). Histograms represent the densitometric mean \pm s.d. $(n=10$ mice $)$.

consistent with an interaction between caspase-2 and these proteins (Fig. 7e,i). Downregulation of caspase-2 before A $\beta$ treatment significantly reduced the translocation of both RhoA and ROCK-II to the spines (Fig. 8).

Taken together, these results suggest that caspase- 2 exerts at least a portion of its effects by controlling the localization, and perhaps the activity of the RhoA/ROCK-II signalling pathway leading to modulation of the actin cytoskeleton in the dendritic spines.

\section{Discussion}

Caspases have are most often thought of in connection with apoptosis. However, several lines of evidence have shown that 
a

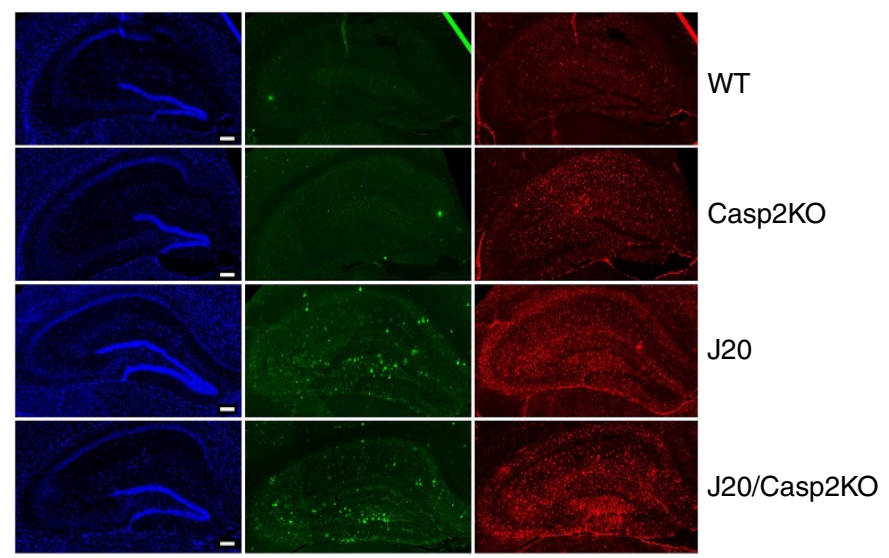

d

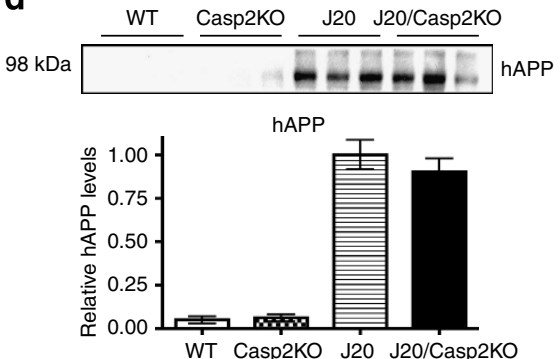

e

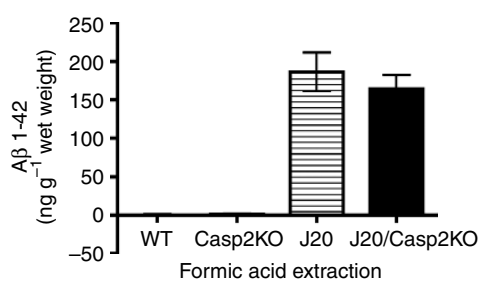

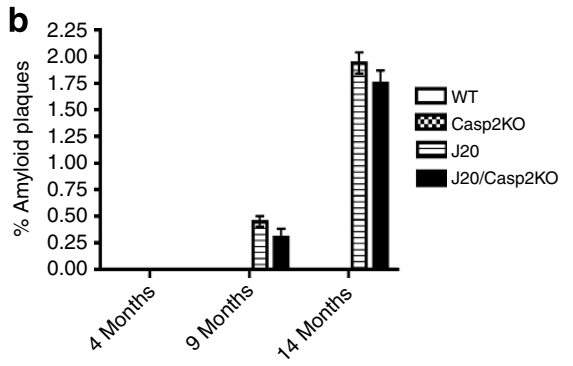
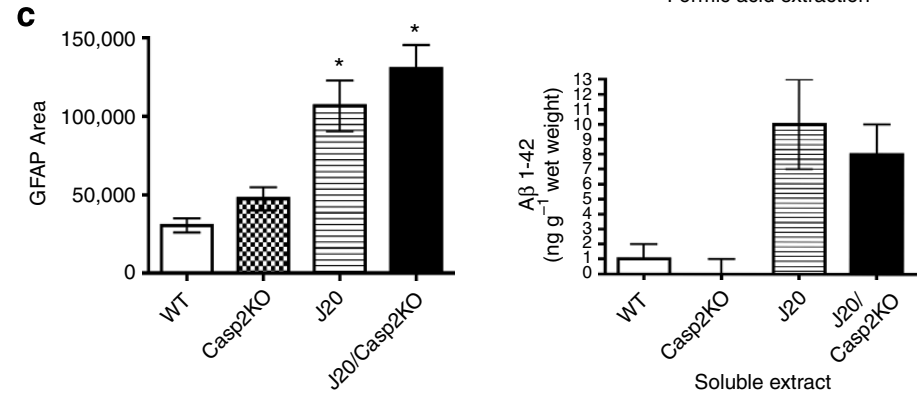

Figure 5 | APP levels, amyloid plaque load and astrogliosis in J20 and J20/Casp2KO mice are similar. At least six mice from each genotype were used to study APP levels and amyloid load. (a) Representative images at 14 months of hippocampal slices stained with DAPI, A $\beta$ (6E10) and GFAP antibodies. (b) Amyloid plaque load in each of the four lines was quantified at 4, 9 and 14 months of age using ImageJ. Data presented as the percentage relative to the total area of the hippocampal slice. (c) GFAP immunopositive areas were quantified using ImageJ. Both J20 and J20/Casp2KO had elevated levels compared with WT or Casp2KO mice. Data are presented as the mean \pm s.d. (Five hippocampal slices per mouse were quantified, ${ }^{\star} P<0.05$, one-way analysis of variance). (d) Representative western blot and densitometric analysis of protein extracts from mouse hippocampus showing similar levels of hAPP in both $\mathrm{J} 20$ and J20. Data presented as the mean \pm s.d. (e) Hippocampal A $\beta_{\mathrm{x}-42}$ levels in both insoluble and soluble fractions were measured by ELISA. There were no significant differences between J2O and Casp2KO mice. Data presented as the mean \pm s.d. ( $n=6$ mice per line).

caspases can also mediate a series of regulatory events in the mature nervous system designed to refine neuronal circuits, such as axon pruning and synapse elimination ${ }^{4-7}$. These processes are tightly regulated presumably by restricting the duration and location of caspase activation ${ }^{32}$. This implies that aberrant and mislocalized activation of caspases in living neurons could have detrimental effects on normal physiology and synaptic functions. Indeed, a number of caspases have been implicated in neurodegenerative diseases, including $\mathrm{AD}^{12-16}$. We previously demonstrated that caspase- 2 was required, with caspase- 3 being dispensable, for the apoptosis-inducing effects of $A \beta$ in cultured hippocampal neurons ${ }^{17,18}$. Naturally, this led us to wonder whether caspase- 2 was also involved in the synaptotoxic effects of $A \beta$.

Using genetic, reverse genetic and pharmacological tools, both in primary neuronal culture and in the intact animal, we show that caspase- 2 is the key regulator of dendritic spine density and morphology in response to $A \beta$. We found that knocking out caspase-2 in the J20 mice, completely prevented many of the agedependent $\mathrm{AD}$-like changes, including the memory deficits in performing the RAWM and the increase in phosphorylated tau protein (pTau). Knocking out caspase- 2 in these mice completely abrogated the changes in spine morphology at an early age (4 months) and blocked the loss of dendritic spine density in older mice (14 months).

It is interesting to note that the protective effects of knocking out caspase-2 in these animals occur without any significant changes in the levels of soluble $A \beta$ or in the extent of plaque load. This raises an intriguing question as to whether $\mathrm{AD}$-like changes in these animal models are due to $A \beta$ or to overexpression of APP. The fact that these animals improve without much change to the $A \beta$ burden suggests the possibility that much of the pathology is $A \beta$-independent. However, this oversimplifies the intimate relationship between $A \beta$ and APP signalling. For example, while $A \beta$ has been demonstrated to be highly toxic to cultured neurons ${ }^{33}$, APP itself appears to be necessary for this toxicity to occur ${ }^{34}$. The induction of APP signalling by its dimerization may in fact be sufficient to trigger neuronal death ${ }^{35}$. However, APP dimerization also leads to increased A $\beta$ production $^{36-38}$ and to the formation of an APP-derived cytosolic toxic fragment, C31 (refs 39-41). Interestingly, blocking the formation of $\mathrm{C} 31$ also causes the reversal of cognitive impairments in J20 mice without affecting the levels of $A \beta^{42,43}$. In our cell culture experiments, it is clear that $A \beta$ causes changes similar to those seen in the mouse and the transduction of these signals requires APP cleavage ${ }^{39-41}$.

In aggregate, these findings suggest a complex interrelation between accumulating $A \beta$ and signalling by APP, its soluble APP ectodomains and its intracellular C-terminal fragments in mediating AD-like changes in animal models. Reducing the levels of any of these components eventually halts this complex signalling machinery allowing for the reversal of the associated cognitive decline $e^{44}$. The relative importance of elements of this 

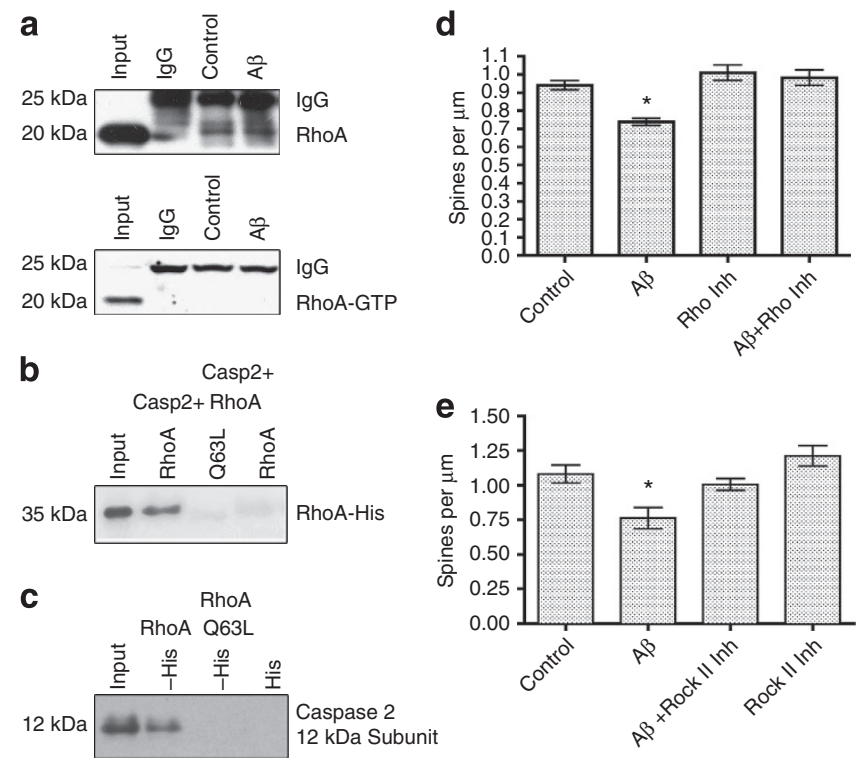

Figure 6 | Inactive RhoA and caspase-2 form a complex.

(a) Immunoprecipitation of caspase-2 in rat hippocampal neuron extracts with showing co-precipitation of RhoA but not RhoA-GTP in both A $\beta$-treated and control cells. (b) RhoA-His but not RhoA(Q63L)-His coprecipitated with recombinant caspase-2 using anti-caspase-2 antibody. (c) Co-precipitation of the caspase-2 $12 \mathrm{kDa}$ subunit with RhoA-His but not with constitutively active RhoA(Q63L)-His using Dynabead His-Tag beads. (d) Synaptosomes from rat hippocampus were pretreated with bVAD-fmk before treatment with $A \beta$ to trap active caspases. Treatment with either $300 \mathrm{nM}$ or $1 \mu \mathrm{M}$ A $\beta$ led to activation of caspase-2. There was no caspase- 2 activation in treated sonicated synaptosomes. (e) Inhibition of RhoA protects against $A \beta$-mediated spine loss. Rat hippocampal primary cultures were pretreated with $0.1 \mu \mathrm{g} \mathrm{ml}^{-1}$ of Rho inhibitor or with PBS for $15 \mathrm{~min}$ before treatment with $300 \mathrm{nM} \mathrm{A} \beta$ for $5 \mathrm{~h}$. A $\beta$ treatment decreased spine number by $20 \%$ in cultures pretreated with PBS compared with control cultures while cultures pretreated with the Rho inhibitor, C3 transferase, showed no spine loss compared with controls. Data presented as the mean \pm s.e.m. ( $n=3$ independent experiments, 150 dendrites per point, ${ }^{\star} P<0.05$, one-way analysis of variance (ANOVA)).

pathway may differ with the stage of the disease and may explain the failure therapeutic strategies designed solely to decrease the levels of $\mathrm{A} \beta$.

The protective effects of knocking out caspase-2 were also recapitulated in a cellular model of $\mathrm{AD}$-related synaptic dysfunction. Both siRNA and chemical inhibitor-mediated approaches to silence or inhibit caspase-2 proved successful in blocking the synaptotoxic effects of $A \beta$ in cultured hippocampal neurons. Consistent with our previous report, silencing of caspase- 3 , caspase- 8 and caspase- 9 individually could not protect cultured neurons against $A \beta$ exposure. Taken together, these results suggest that caspase- 2 is a key factor in a novel mechanism regulating dendritic spine architecture and neural function that is ultimately mirrored in the behavioural tasks.

These results are at variance with two recent reports implicating caspase- 3 in the modulation of spine dynamics in similar mouse models ${ }^{45,46}$. It should be noted that the first of these studies relied on the detection of cleaved caspase- 3 as a measure of activity. However, the cleavage of caspase-3 does not necessarily indicate that it is active in cells. Indeed, caspases are subject to regulation by a wide array of IAPs (inhibitor of apoptosis) and other regulatory proteins ${ }^{18,47}$. In the absence of specific intracellular reporters, in situ bVAD trapping of active caspases $^{48}$ remains the most reliable way to confirm that a given caspase is active. Furthermore, the exclusive reliance on pseudosubstrate inhibitors in that study precludes drawing any definitive conclusion as to the involvement of a specific caspase, as these inhibitors lack the required specificity ${ }^{49}$. The second report attempts to overcome several of these limitations by using the BIR domain of IAP proteins to specifically block caspase- 3 activity. Although this domain does not affect caspase- 2 activity, the analysis of the data is confounded by the fact that the BIR domain can also directly interact with cytoskeletal elements and block cytokinesis, as demonstrated in C. elegans ${ }^{50}$. Furthermore, the XIAP ring domain can also interact with Rho-regulatory protein, RhoGDI and promote the assembly of actin, a key component of dendritic spines ${ }^{51}$. Thus, it cannot be ruled out that the BIR-mediated protective effect is due to action on the actin cytoskeleton rather than on inhibition of caspase-3. The fact that depleting the protein levels of caspase-3 through RNAi in our studies fails to confer any protection against $A \beta$ in neurons argues against a central role for caspase- 3 in this process.

The exact mechanism by which caspase- 2 mediates the synaptotoxic effects of $A \beta$ is an intriguing one and our results offer several important clues. Both caspase- 2 and RhoA appear to reside outside of the spine head in the form of an inactive complex under normal conditions. This is supported by the fact that only inactive RhoA co-precipitates with caspase- 2 and by the fact that we observe very little colocalization between them and the synaptic marker, PSD95. However, exposure to A $\beta$ results in the activation of both caspase- 2 and RhoA and the dissociation of the complex (Fig. 6). In their active form, both RhoA and caspase- 2 appear to enter the spine head, demonstrated by the fact that there is a significant increase in their co-localization with PSD95. One interesting observation is that downregulating caspase-2 is not sufficient for the translocation of RhoA to spines, suggesting that another anchor may be preventing this occurrence. A possible candidate for this role is the Rho-GDP dissociation inhibitor (RhoGDI), which sequesters Rho-GTPases from the membrane and prevents their interaction with guanine exchange factors. An interesting line for future investigations is to determine if both caspase-2 and Rho-GDI complex with RhoAGDP at the same time or whether their binding is mutually exclusive.

Once active RhoA and caspase- 2 enter the spine head, they can affect the activity of one or more effectors to drive the collapse of the spines. One immediate candidate is the Rho effector ROCKII, which has previously been shown to be activated by caspase-2mediated cleavage ${ }^{28}$, and which we show to be cleaved in neurons treated with $A \beta$.

In summary, our results clearly demonstrate that caspase- 2 is an important mediator of $\mathrm{A} \beta$ synaptotoxicity in culture and in the intact animal. As caspase- 2 expression is elevated in $\mathrm{AD}$ brain $^{52,53}$, it is possible that inhibition of caspase-2, especially early in the course of $\mathrm{AD}$, might prevent synaptic changes and memory deficits. The fact that we can protect neurons against $A \beta$ with only a partial downregulation of caspase- 2 suggests that therapeutic protection might be achieved without blocking critical actions of caspase- 2 in other systems.

\section{Methods}

Transgenic mice. All animal studies were performed according to protocols examined and approved by the Animal Use and Care Committee of Columbia University. The J20 transgenic mouse line expresses a mutated human APP (hAPP: K670N/M671L and V717F) under the control of the platelet-derived growth factor promoter ${ }^{19,54}$. In our studies, we have used a J20 mouse line crossed to a C57BL/6 background (courtesy of Dr S-D Yan). Caspase-2 knockout (Casp2KO) mice ${ }^{22}$ were also back-crossed to a C57BL/6 background. Double transgenic mice were obtained by crossing hemizygous transgenic mice J20 with Casp2KO mice. The first generation was crossed again with Casp2KO mice to generate the dual transgenic/null J20/Casp2KO mice. Transgenic mice were genotyped by PCR using 
a

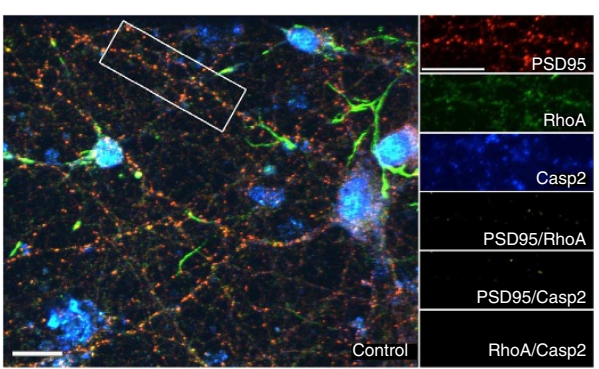

b

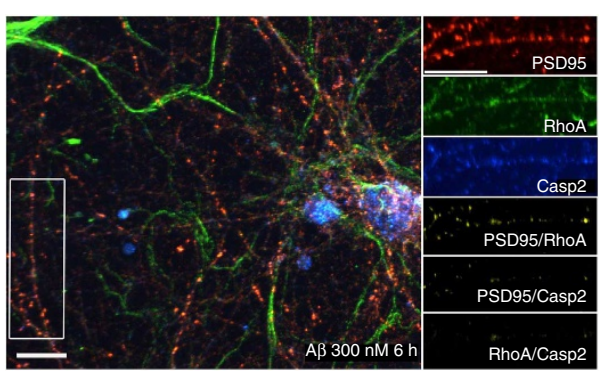

c

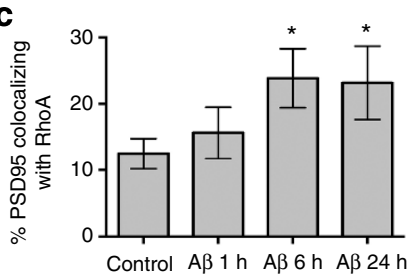

d

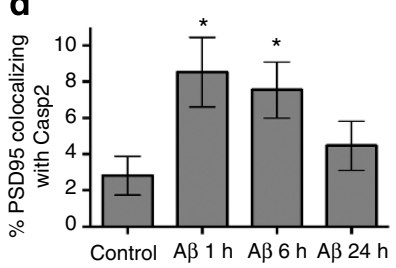

e

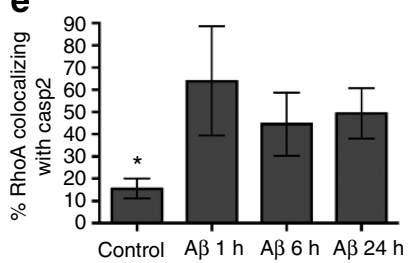

f

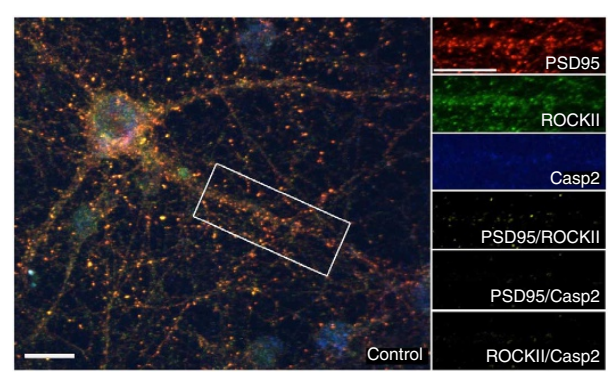

h

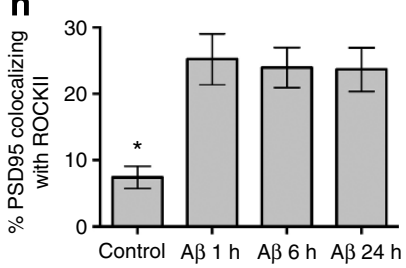

g

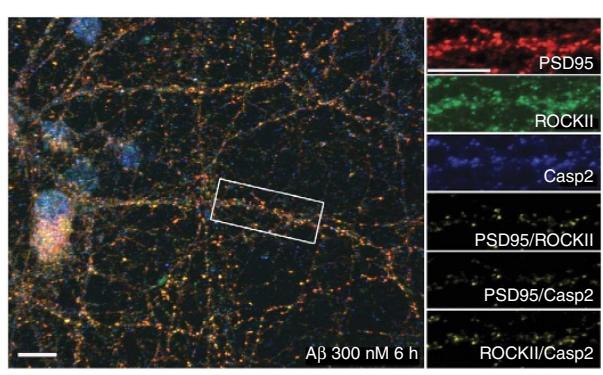

i

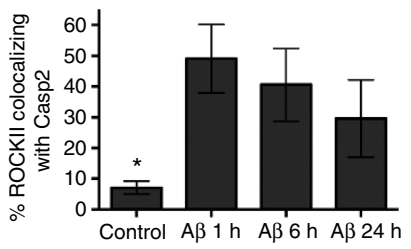

Figure 7 | RhoA, ROCK-II and caspase-2 translocate to spines following A $\boldsymbol{\beta}$ treatment. (a) Representative confocal images of vehicle-treated cultures stained for PSD95 (Red), RhoA (green) and caspase-2 (blue). Higher magnification examination of a dendritic segment revealed that both RhoA and caspase-2 were present mainly in dendritic shafts. Colocalization of proteins is represented in yellow (analysed with Volocity software; boxed areas showing enlarged dendritic segments. Scale bar, $10 \mu \mathrm{m}$ ). (b) Representative confocal images of culture hippocampal neurons treated for $6 \mathrm{~h}$ with $A \beta$ $300 \mathrm{nM}$ and stained for PSD95 (Red), RhoA (green) and caspase-2 (blue). (c) Quantification of colocalization of RhoA with PSD95, represented as the percentage of PSD95 puncta colocalized with RhoA. Data presented as the mean \pm s.e.m. ( $n=3$ independent experiments, ${ }^{\star} P<0.05$, one-way analysis of variance (ANOVA)). (d) Quantification of colocalization of caspase-2 with PSD95, represented as the percentage of the PSD95 puncta colocalized with RhoA. Data presented as the mean \pm s.e.m. ( $n=3$ independent experiments, ${ }^{\star} P<0.05$, one-way ANOVA). (e) Quantification of colocalization of caspase- 2 with RhoA, represented as the percentage of the RhoA puncta colocalized with caspase-2. Data presented as the mean \pm s.e.m. $(n=3$ independent experiments, ${ }^{\star} P<0.05$, one-way ANOVA). (f) Representative confocal image of an untreated hippocampal neuron stained for PSD95 (Red), ROCK-II (green) and caspase-2 (blue). Higher magnification of a dendritic segment revealed that both ROCK-II and caspase-2 were present mainly in dendritic shafts. Colocalization of proteins is represented in yellow (analysed with Volocity software; boxed areas showing enlarged dendritic segments. Scale bar, $10 \mu \mathrm{m})$. (g) Representative confocal image of a treated cultured hippocampal neuron (A $\beta, 300 \mathrm{nM}$ ) stained for PSD95 (Red), ROCK-II (green) and caspase2 (blue). (h) Quantification of colocalization of ROCK-II with PSD95, represented as the percentage of PSD95 puncta that colocalized with ROCK-II. Data presented as the mean \pm s.e.m. ( $n=3$ independent experiments, ${ }^{\star} P<0.05$, one-way ANOVA). (i) Quantification of colocalization of caspase- 2 with ROCKII, represented as the percentage of ROCK-II puncta colocalized with caspase-2. Data presented as the mean \pm s.e.m. $(n=3$ independent experiments, ${ }^{\star} P<0.05$, one-way ANOVA).

oligonucleotides for human APP and caspase- 2 with PuRe Taq PCR beads (Amersham).

Male mice were divided into the following groups: WT, caspase-2 knockout (Casp2KO), J20 and J20/Casp2KO. Mice were tested blindly in the following experimental sequence: behaviour, biochemistry and histology. The complete sequence of behavioural tests was performed in the same mice at 4, 9 and 14 months of age. For each of the age groups, the complete sequence of behavioural tests required $\sim 3$ weeks and consisted of the RAWM test, and visible platform test.
Immediately after behavioural testing at 14 month of age, mice were killed for DiOlistic labelling, biochemical and histological studies. Female mice were used only for the DiOlistic labelling, the biochemical, and histological studies and not for the behavioural studies.

Radial-arm water maze. The task has been described previously ${ }^{55}$. Each day of testing included four consecutive trials (A1-A4) and a fifth trial (R) $30 \mathrm{~min}$ after 

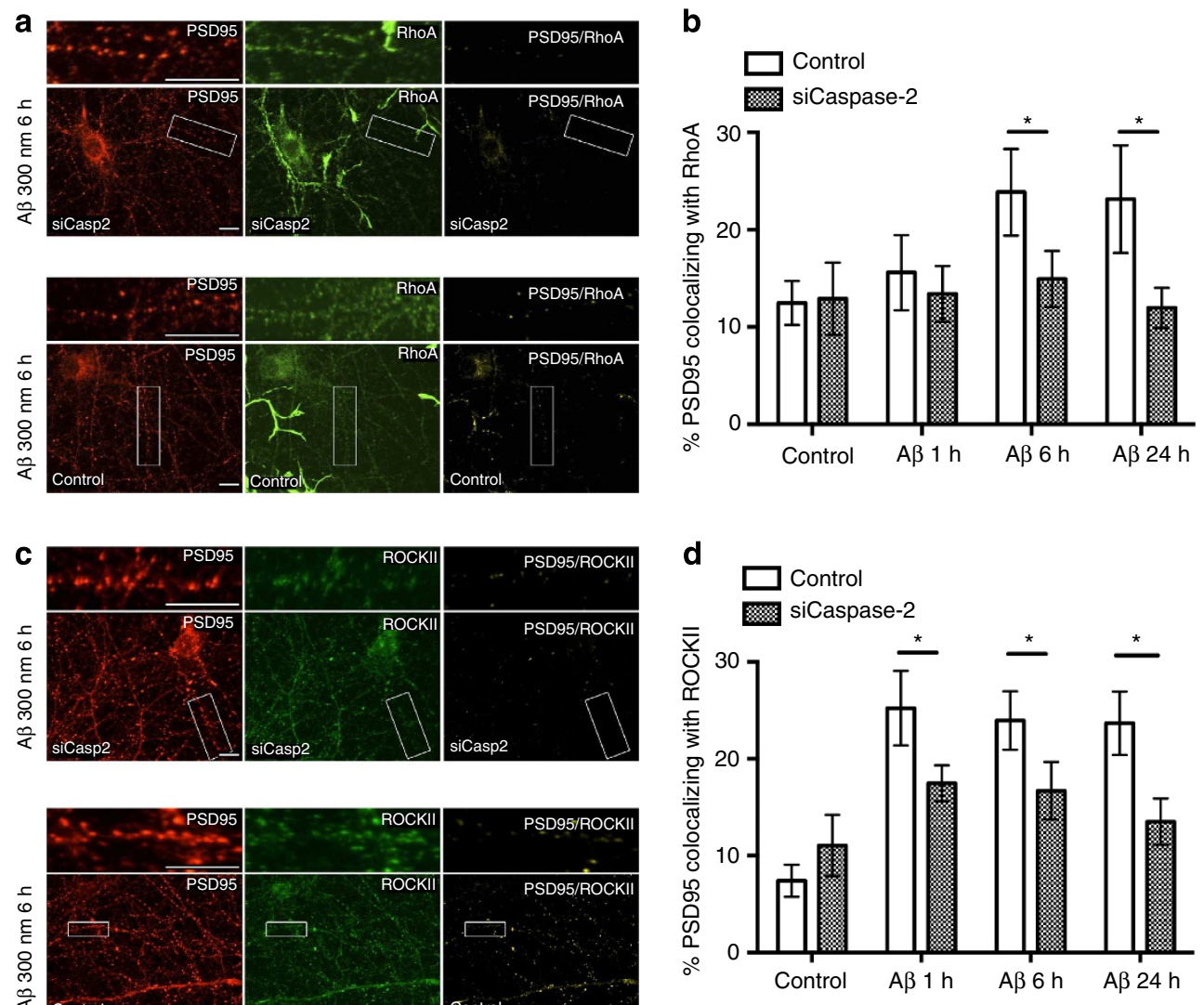

Figure 8 | A -induced RhoA and ROCK-II translocation to dendritic spines is blocked by downregulation of caspase-2. (a) Representative confocal images of hippocampal cultures stained for PSD95 (Red) and RhoA (green), pretreated with siCasp2 or with vehicle (control) and treated for $6 \mathrm{~h}$ with $300 \mathrm{nM}$ A $\beta$. Colocalization of proteins is represented in yellow (analysed with Volocity software; boxed areas showing enlarged dendritic segments. Scale bar, $10 \mu \mathrm{m}$ ). (b) Quantification of colocalization of RhoA with PSD95, represented as the percentage of PSD95 puncta colocalized with RhoA. Colocalization was measured before treatment with $A \beta$ and at 1,6 and $24 \mathrm{~h}$ after treatment. Data presented as the mean \pm s.e.m. ( $n=3$ independent experiments, ${ }^{\star} P<0.05$, one-way analysis of variance (ANOVA)). (c) Representative confocal image of a hippocampal neuron stained for PSD95 (Red) and ROCK-II (green) pretreated with siCasp2 or with vehicle and treated for $6 \mathrm{~h}$ with $300 \mathrm{nM} \mathrm{A \beta}$. Colocalization of proteins is represented in yellow (analysed with Volocity software; boxed areas showing enlarged dendritic segments. Scale bar, $10 \mu \mathrm{m}$ ). (d) Quantification of colocalization of ROCK-II with PSD95, represented as the percentage of PSD95 puncta colocalized with ROCK-II. Colocalization was measured before treatment with A $\beta$ and at 1,6 and $24 \mathrm{~h}$ after treatment. Data presented as the mean \pm s.e.m. $\left(n=3\right.$ independent experiments, ${ }^{\star} P<0.05$, one-way ANOVA).

the fourth trial. The first four trials tested acquisition of the task, while the fifth trial tested retention memory (short-term memory). Each trial lasted $1 \mathrm{~min}$. Errors were counted when the mouse went to an arm without platform or took more than $20 \mathrm{~s}$ to enter any arm of the maze. The number of errors in each trial in the last 3 days of testing was averaged and used for statistical analysis.

DiOlistic labelling. DiOlistic labelling was performed as previously described ${ }^{56}$. Briefly, mice were anaesthetized then fixed with $4 \%$ paraformaldehyde by transcardiac perfusion. Their brains were removed and sectioned coronally $(300 \mu \mathrm{M})$ using a vibratome. Tissue sections were subsequently shot with DiIcoated particles using the Helios gene gun system. Hippocampal neurons were visualized using a spinning Disk Confocal Microscope and images were acquired with Perkin Elmer Volocity Software. Spines were counted manually in a doubleblinded manner. NeuronStudio software was use to analyse the spine subtype populations.

Histological studies. Immunolabeling was done on $30 \mu \mathrm{m}$ brain sections from the four genotypes. Staining was done using the anti $A \beta$ antibody $6 \mathrm{E} 10$ (Covance), $\mathrm{A} \beta_{1-42}$ (Abbiotech), Pan-Axonal Neurofilament SMI-312 (Covance), GFAP (Cell Signaling), anti microglia Iba-1 (Abcam). Plaque load and astrocytosis were quantified using ImageJ. Total plaque area was expressed as a percentage of the total hippocampal area. Astrocytosis was expressed as total area units.

ELISA and western blot analysis. Whole-frozen hippocampi were homogenized using a Teflon dounce homogenizer first in $150 \mathrm{mg} \mathrm{ml}^{-1}$ in $2 \% \mathrm{w} / \mathrm{v}$ sodium dodecyl sulphate (SDS) in water with protease inhibitors (Roche) to recover soluble amyloid. The sample was then centrifuged and the pellet homogenized in $70 \%$ formic acid in water to obtain the insoluble amyloid fraction. Homogenates were spun at $100,000 \mathrm{~g}$ for $1 \mathrm{~h}$. Supernates were diluted in buffer EC $(0.02 \mathrm{M}$ sodium phosphate, $\mathrm{pH} 7.0,0.2 \mathrm{mM}$ EDTA, $0.4 \mathrm{M} \mathrm{NaCl}, 0.2 \%$ bovine serum albumin, $0.05 \%$ CHAPS) on ice. To obtain the soluble amyloid fraction and protein for western blotting, frozen hippocampi were homogenized using a Teflon dounce homogenizer in cell lysis buffer (Cell Signaling), sonicated briefly and centrifuged at 14,000 r.p.m. for $10 \mathrm{~min}$. $\mathrm{A} \beta_{\mathrm{X}-42}$ was measured by ELISA (Covance) following the manufacturers protocol. Immunoblotting was performed using the following polyclonal antibodies C-terminal APP (Calbiochem), caspase-2 (Abcam), PSD95 (Cell Signaling), Synapsin-1 (Chemicon) and ERK1 (Santa Cruz) and the monoclonal antibody for phospho-tau PHF13 (Cell Signaling). The caspase-2 polyclonal antibody was provided by the Troy laboratory ${ }^{57}$. Representative complete gels can be found in the Supplementary Information (Supplementary Fig. S7).

Immunofluorescence and microscopy. Cells were fixed in paraformaldehyde (4\%) in PBS for 10 min. Permeabilization was performed using $0.4 \%$ triton in PBS for $20 \mathrm{~min}$. Cells were stained using the following antibodies PSD95 (Cell Signaling), Synapsin-1 (Chemicon) and caspase-2 (Millipore). Primary cultures were visualized using a spinning disk confocal microscope and images were acquired with Perkin Elmer Volocity software.

Data analysis and statistics. Immunoblotting, primary cultures of spine density, behavioural ELISA analysis and plaque burden analyses were performed with a two-tailed Student's $t$-test. Mouse spine density data were analysed by two-way analysis of variance with age and genotype as independent variables. In all cases, $P<0.05$ was considered to be statistically significant. 


\section{References}

1. McGowan, E., Eriksen, J. \& Hutton, M. A decade of modeling Alzheimer's disease in transgenic mice. Trend genet. 22, 281-289 (2006).

2. McLarnon, J. G. \& Ryu, J. K. Relevance of abeta1-42 intrahippocampal injection as an animal model of inflamed Alzheimer's disease brain. Curr. Alzheimer. Res. 5, 475-480 (2008).

3. Vitolo, O. et al. Protection against beta-amyloid induced abnormal synaptic function and cell death by Ginkgolide J. Neurobiol. Aging 30, 257-265 (2009).

4. Kuo, C. T., Zhu, S., Younger, S., Jan, L. Y. \& Jan, Y. N. Identification of E2/E3 ubiquitinating enzymes and caspase activity regulating Drosophila sensory neuron dendrite pruning. Neuron 51, 283-290 (2006).

5. Williams, D. W., Kondo, S., Krzyzanowska, A., Hiromi, Y. \& Truman, J. W. Local caspase activity directs engulfment of dendrites during pruning. Nat. Neurosci. 9, 1234-1236 (2006)

6. Nikolaev, A., McLaughlin, T., O’Leary, D. D. \& Tessier-Lavigne, M. APP binds DR6 to trigger axon pruning and neuron death via distinct caspases. Nature 457, 981-989 (2009).

7. Ohsawa, S. et al. Maturation of the olfactory sensory neurons by Apaf-1/ caspase-9-mediated caspase activity. Proc. Natl Acad. Sci. USA 107, 13366-13371 (2010)

8. Li, Z. et al. Caspase-3 activation via mitochondria is required for long-term depression and AMPA receptor internalization. Cell 141, 859-871 (2010).

9. Jiao, S. \& Li, Z. Nonapoptotic function of BAD and BAX in long-term depression of synaptic transmission. Neuron 70, 758-772 (2011).

10. Masliah, E. et al. Synaptic and neuritic alterations during the progression of Alzheimer's disease. Neurosci. Lett. 174, 67-72 (1994).

11. Ingelsson, M. et al. Early Abeta accumulation and progressive synaptic loss, gliosis, and tangle formation in AD brain. Neurology 62, 925-931 (2004).

12. Allen, J. W., Eldadah, B. A., Huang, X., Knoblach, S. M. \& Faden, A. I. Multiple caspases are involved in beta-amyloid-induced neuronal apoptosis. J. Neurosci. Res. 65, 45-53 (2001).

13. Sheng, J. G. et al. Neuronal DNA damage correlates with overexpression of interleukin-1beta converting enzyme in APPV717F mice. Neurobiol. Aging 22, 895-902 (2001)

14. Pellegrini, L., Passer, B. J., Tabaton, M., Ganjei, J. K. \& D’Adamio, L. Alternative, non-secretase processing of Alzheimer's beta-amyloid precursor protein during apoptosis by caspase-6 and -8. J. Biol. Chem. 274, 21011-21016 (1999).

15. LeBlanc, A., Liu, H., Goodyer, C., Bergeron, C. \& Hammond, J. Caspase-6 role in apoptosis of human neurons, amyloidogenesis, and Alzheimer's disease. J. Biol. Chem. 274, 23426-23436 (1999).

16. Guo, H. et al. Active caspase- 6 and caspase-6-cleaved tau in neuropil threads, neuritic plaques, and neurofibrillary tangles of Alzheimer's disease. Am. J. Pathol. 165, 523-531 (2004).

17. Troy, C. M. et al. Caspase- 2 mediates neuronal cell death induced by betaamyloid. J. Neurosci. 20, 1386-1392 (2000).

18. Troy, C. M. et al. Death in the balance: alternative participation of the caspase-2 and -9 pathways in neuronal death induced by nerve growth factor deprivation. J. Neurosci. 21, 5007-5016 (2001).

19. Mucke, L. et al. High-level neuronal expression of abeta 1-42 in wild-type human amyloid protein precursor transgenic mice: synaptotoxicity without plaque formation. J. Neurosci. 20, 4050-4058 (2000).

20. Tashiro, A. \& Yuste, R. Regulation of dendritic spine motility and stability by Racl and Rho kinase: evidence for two forms of spine motility. Mol. Cell Neurosci. 26, 429-440 (2004).

21. Tashiro, A., Minden, A. \& Yuste, R. Regulation of dendritic spine morphology by the rho family of small GTPases: antagonistic roles of Rac and Rho. Cereb. Cortex. 10, 927-938 (2000).

22. Bergeron, L. et al. Defects in regulation of apoptosis in caspase-2-deficient mice. Genes Dev. 12, 1304-1314 (1998).

23. Masliah, E., Mallory, M., Hansen, L., DeTeresa, R. \& Terry, R. D. Quantitative synaptic alterations in the human neocortex during normal aging. Neurology 43, 192-197 (1993).

24. Masliah, E. et al. Comparison of neurodegenerative pathology in transgenic mice overexpressing V717F beta-amyloid precursor protein and Alzheimer's disease. J. Neurosci. 16, 5795-5811 (1996).

25. Grathwohl, S. A. et al. Formation and maintenance of Alzheimer's disease betaamyloid plaques in the absence of microglia. Nat. Neurosci. 12, 1361-1363 (2009).

26. Tizon, B., Ribe, E. M., Mi, W., Troy, C. M. \& Levy, E. Cystatin C protects neuronal cells from amyloid-beta-induced toxicity. J. Alzheimer. Dis. 19, 885-894 (2010)

27. Kong, J. Y. \& Rabkin, S. W. The association between RhoB and caspase-2: changes with lovastatin-induced apoptosis. Biochem. Cell Biol. 83, 608-619 (2005).

28. Sapet, C. et al. Thrombin-induced endothelial microparticle generation: identification of a novel pathway involving ROCK-II activation by caspase- 2 . Blood 108, 1868-1876 (2006).
29. Sebbagh, M., Hamelin, J., Bertoglio, J., Solary, E. \& Breard, J. Direct cleavage of ROCK II by granzyme B induces target cell membrane blebbing in a caspaseindependent manner. J. Exp. Med. 201, 465-471 (2005).

30. Huesa, G. et al. Altered distribution of RhoA in Alzheimer's disease and AbetaPP overexpressing mice. J. Alzheimer. Dis. 19, 37-56 (2010).

31. Schubert, V., Da Silva, J. S. \& Dotti, C. G. Localized recruitment and activation of RhoA underlies dendritic spine morphology in a glutamate receptordependent manner. J. Cell Biol. 172, 453-467 (2006).

32. Hyman, B. T. Caspase activation without apoptosis: insight into Abeta initiation of neurodegeneration. Nat. Neurosci. 14, 5-6 (2011).

33. Cavallucci, V., D'Amelio, M. \& Cecconi, F. Abeta toxicity in Alzheimer's disease. Mol. Neurobiol. 45, 366-378 (2012).

34. Shaked, G. M. et al. Abeta induces cell death by direct interaction with its cognate extracellular domain on APP (APP 597-624). FASEB J. 20, 1254-1256 (2006).

35. Rohn, T., Ivins, K., Bahr, B., Cotman, C. \& Cribbs, D. A monoclonal antibody to amyloid precursor protein induces neuronal apoptosis. J. Neurochem. 74, 2331-2342 (2000).

36. Lefort, R., Pozueta, J. \& Shelanski, M. Cross-linking of cell surface amyloid precursor protein leads to increased beta-amyloid peptide production in hippocampal neurons: implications for Alzheimer's disease. J. Neurosci. 32, 10674-10685 (2012).

37. Scheuermann, S. et al. Homodimerization of amyloid precursor protein and its implication in the amyloidogenic pathway of Alzheimer's disease. J. Biol. Chem. 276, 33923-33929 (2001).

38. Munter, L.-M. et al. GxxxG motifs within the amyloid precursor protein transmembrane sequence are critical for the etiology of Abeta42. EMBO J. 26, 1702-1712 (2007).

39. Lu, D. C. et al. A second cytotoxic proteolytic peptide derived from amyloid beta-protein precursor. Nat. Med. 6, 397-404 (2000)

40. Lu, D. C., Shaked, G. M., Masliah, E., Bredesen, D. E. \& Koo, E. H. Amyloid beta protein toxicity mediated by the formation of amyloid-beta protein precursor complexes. Ann. Neurol. 54, 781-789 (2003).

41. Lu, D. C., Soriano, S., Bredesen, D. E. \& Koo, E. H. Caspase cleavage of the amyloid precursor protein modulates amyloid beta-protein toxicity. J. Neurochem. 87, 733-741 (2003).

42. Galvan, V. et al. Reversal of Alzheimer's-like pathology and behavior in human APP transgenic mice by mutation of Asp664. Proc. Natl Acad. Sci. USA 103, 7130-7135 (2006).

43. Saganich, M. J. et al. Deficits in synaptic transmission and learning in amyloid precursor protein (APP) transgenic mice require C-terminal cleavage of APP. J. Neurosci. 26, 13428-13436 (2006).

44. Melnikova, T. et al. Reversible pathologic and cognitive phenotypes in an inducible model of Alzheimer-amyloidosis. J. Neurosci. 33, 3765-3779 (2013).

45. D'Amelio, M. et al. Caspase-3 triggers early synaptic dysfunction in a mouse model of Alzheimer's disease. Nat. Neurosci. 14, 69-76 (2011).

46. Jo, J. et al. Abeta(1-42) inhibition of LTP is mediated by a signaling pathway involving caspase-3, Aktl and GSK-3beta. Nat. Neurosci. 14, 545-547 (2011).

47. Troy, C. M. \& Shelanski, M. L. Caspase-2 redux. Cell Death Differ. 10, 101-107 (2003).

48. Tu, S. et al. In situ trapping of activated initiator caspases reveals a role for caspase-2 in heat shock-induced apoptosis. Nat. Cell Biol. 8, 72-77 (2006).

49. McStay, G. P., Salvesen, G. S. \& Green, D. R. Overlapping cleavage motif selectivity of caspases: implications for analysis of apoptotic pathways. Cell Death Differ. 15, 322-331 (2008)

50. Fraser, A. G., James, C., Evan, G. I. \& Hengartner, M. O. Caenorhabditis elegans inhibitor of apoptosis protein (IAP) homologue BIR-1 plays a conserved role in cytokinesis. Curr. Biol. 9, 292-301 (1999).

51. Liu, J. et al. X-linked inhibitor of apoptosis protein (XIAP) mediates cancer cell motility via Rho GDP dissociation inhibitor (RhoGDI)-dependent regulation of the cytoskeleton. J. Biol. Chem. 286, 15630-15640 (2011).

52. Shimohama, S., Tanino, H. \& Fujimoto, S. Changes in caspase expression in Alzheimer's disease: comparison with development and aging. Biochem. Biophys. Res. Commun. 256, 381-384 (1999).

53. Pompl, P. N. et al. Caspase gene expression in the brain as a function of the clinical progression of Alzheimer disease. Arch. Neurol. 60, 369-376 (2003).

54. Abrahamson, E. et al. Caspase inhibition therapy abolishes brain traumainduced increases in Abeta peptide: implications for clinical outcome. Exp. Neurol. 197, 437-450 (2006)

55. Trinchese, F. et al. Progressive age-related development of Alzheimer-like pathology in APP/PS1 mice. Ann. Neurol. 55, 801-814 (2004).

56. Moolman, D. L., Vitolo, O. V., Vonsattel, J.-P. G. \& Shelanski, M. L. Dendrite and dendritic spine alterations in Alzheimer models. J. Neurocytol. 33, 377-387 (2004).

57. Troy, C., Stefanis, L., Greene, L. \& Shelanski, M. Mechanisms of neuronal degeneration: a final common pathway? Adv. Neurol. 72, 103-111 (1997). 


\section{Acknowledgements}

This work was supported in part by Grants NIHAG08702 and NS15076, the Wallace Foundation for Research and the Taub Foundation.

\section{Author contributions}

J.P. and R.L. contributed equally to this work. All authors contributed to the design of the experiments. J.P., R.L. and E.M.R. performed the experiments. J.P., R.L. and M.S. wrote the paper. O.A. analysed and interpreted the behavioural data. All authors discussed the results and implications and commented on the manuscript at all stages.

\section{Additional information}

Supplementary Information accompanies this paper at http://www.nature.com/ naturecommunications

Competing financial interests: The authors declare no competing financial interests.

Reprints and permission information is available online at http://npg.nature.com/ reprintsandpermissions/

How to cite this article: Pozueta, J. et al. Caspase-2 is required for dendritic spine and behavioural alterations in J20 APP transgenic mice. Nat. Commun. 4:1939 doi: 10.1038/ ncomms2927 (2013). 